\title{
Extending a structural model of somatization to South Koreans: Cultural values,somatization tendency, and the presentation of depressive symptoms
}

\author{
Xiaolu Zhou ${ }^{1}$, Seongho $\mathrm{Min}^{2}$, Jiahong $\mathrm{Sun}^{3}$, See Joo Kim ${ }^{4}$, Joung-sook Ahn ${ }^{2}$, Yunshi Peng ${ }^{5}$, Samuel \\ Noh $^{6,7}$, and Andrew G. Ryder ${ }^{3,8}$ \\ ${ }^{1}$ Shanghai Normal University \\ ${ }^{2}$ Yonsei University Wonju School of Medicine \\ ${ }^{3}$ Concordia University \\ ${ }^{4}$ Yonsei University College of Medicine, \\ ${ }^{5}$ Human Normal University \\ ${ }^{6}$ Centre for Addiction and Mental Health \\ ${ }^{7}$ University of Toronto \\ ${ }^{8}$ Jewish General Hospital
}

\begin{abstract}
Background: Somatization refers to the tendency to emphasize somatic symptoms when experiencing a psychiatric disturbance. This tendency has been widely reported in patients from East Asian cultural contexts suffering from depression. Recent research in two Chinese samples have demonstrated that the local cultural script for depression, involving two aspects- the experience and expression of distress (EED) and conceptualization and communication of distress (CCD) — can be evoked to help explain somatization. Given the beliefs and practices broadly shared across Chinese and South Korean cultural contexts, the current study seeks to replicate this explanatory model in South Koreans.

Methods: Our sample included 209 psychiatric outpatients from Seoul and Wonju, South Korea. Self report questionnaires were used to assess somatization tendency, adherence to traditional values, and psychological and somatic symptoms of depression.

Results: Results from SEM showed that the EED and CCD factors of somatization tendency were differently associated with cultural values and somatic symptoms, replicating our previous findings in Chinese outpatients.

Limitations: The reliance on a brief self-report measure of somatization tendency, not originally designed to assess separate EED and CCD factors, highlights the need for measurement tools for the assessment of cultural scripts in cross-cultural depression research.

Conclusions: The replication of the Chinese structural model of somatization in South Korea lends empirical support to the view that somatization can be understood as the consequence of specific cultural scripts. These scripts involve the experience and expression of distress as well as culturally meaningful ways in which this distress is conceptualized and communicated to others.
\end{abstract}

\section{Introduction}

Somatization refers to the tendency to emphasize somatic symptoms when experiencing a psychological disorder (Kirmayer and Robbins, 1991). Somatic symptoms are often reported in the context of depression across cultural contexts (Simon et al., 1999). However, patients from some cultural backgrounds are more likely to report bodily disturbances than others (Parker et al., 2001; Ryder et al., 2008). Given the prominence of somatic symptoms in depression, we contend that a fine-tuned understanding of somatization will not only benefit the diagnosis but also the treatment of depression in clinical practice.

Kleinman $(1982,1986)$, in his pioneering work in China, found that Chinese psychiatric patients strongly emphasized somatic symptoms of depression. This observation has since been empiri cally supported in several cross-cultural studies (Parker et al., 2001; Ryder et al., 2008; Zhou et al., 2011; Dere et al., 2013) comparing Chinese and 'Western' participants. Ryder and Chentsova-Dutton (2012) proposed that cultural scripts for distress and normative assumptions about symptoms and syndromes in a given cultural context might help shape the experience and expression of depression. They further argued that the emphasis on somatic symptoms could be understood as the consequence of a specific local cultural script of depression. This script is thought to involve at least two aspects: one involving the experience 
and expression of distress (EED); and one involving the conceptualization and communication of distress (CCD).

In a recent study of Chinese university students and depressed Chinese outpatients Zhou et al. (in press) confirmed that an established measure of somatization tendency is divisible into two correlated factors corresponding to these two aspects. Moreover, they found that these factors are differentially associated with levels of somatic symptom reporting and traditional values: EED was primarily associated with somatic symptom reporting and CCD was primarily associated with endorsement of traditional Chinese values.

Although the literature on culture and somatization has emphasized the Chinese case, research in recent years has turned to other East Asian cultural contexts. A number of studies focusing on Korean immigrant communities in the U.S. have demonstrated that somatic symptoms are frequently reported among migrants from South Korea (Keyes and Ryff, 2003; Kim, 2002; Kim et al., 1999; Pang, 1998, 2000; Park and Bernstein, 2008; Yoo and Skovhot, 2001). Furthermore, somatization is an important part of a depression-like culture-bound syndrome, Hwa-Byung (Lin et al., 1992; Suh, 2013). Hwa-Byung, literally meaning anger syndrome, is a chronic psycho somatic condition where long-standing but incomplete suppression of negative affects are manifested through physical disturbances, such as insomnia, fatigue, heart palpitations, and so on (Park et al., 2001). Given the frequent and common experiences of somatic symptoms in depression and Hwa-Byung, some South Koreans use these terms interchangeably (Pang, 1998). As in China, the South Korean cultural script for depression may similarly involve more somatic responses to psychosocial stressors (i.e., EED) and a somatic emphasis in the conceptualization of distress and the communication of this distress to others (i.e., $\mathrm{CCD}$ ).

What are the cultural bases for EED and CCD? The EED aspect of somatization may find its roots in traditional Chinese medicine, which shapes beliefs about the body and mind in China and other East Asian societies. In traditional Chinese medicine, experiences of emotions and organ functioning are closely connected and mutually influential. For ex-

Abbreviations: $\mathrm{CCD}$, conceptualization and communication of distress; EED, experience and expression of distress; SEM structural equation modeling

${ }^{*}$ Corresponding author at: Department of Management, Education College, Shanghai Normal University, 100 Guilin Rd., Shanghai 200234,China. Tel. +1 8621 64324192;fax: +86 2164322450.

** Corresponding author at:Department of Psychology (PY139-3), Concordia University,7141 Sherbrooke St. W.,Montréal,Québec H4B1R6,Canada.Tel.: +1 5148482424 x 5379; fax: +1 514848 4523. E-mail addresses: zhouziaolu@ @hnu.edu.cn (X. Zhou), andrew.ryder@concordia.ca (A.G.Ryder). ample, experiencing negative affect or depression is associated with dysfunction of the liver and kidney (Kim and Rhi, 1976). Extreme psychosocial stress can elevate the seriousness of physical disturbances, fostering a sense that one's body is in danger-as in local expressions such as, "my blood will dry up" (Pang, 1998).

The CCD aspect of somatization, on the other hand, emphasizes cultural values and help-seeking behavior. In hierarchical societies, people from lower socioeconomic strata tend to suppress negative affect in order to maintain a harmonious environment in the family or society (Kim, 1973). Reporting physical discomfort, therefore, may present a better way of communicating negative feelings and distress than to directly express one's displeasure (Pang, 1998). In addition, discussing somatic instead of psychological distress may culturally appropriate way to seek help. For example, South Koreans showed more passive attitudes toward helpseeking from mental health professionals compared to Americans (Yoo, 2001; Park et al., 2013). In fact, South Koreans who suffer from mental health problems will often resort to physician's consultation, herbal medicine, healthy foods, prescription drugs, and so on, for relief (Kim, 1999).

Our brief overview of the literature suggests that at least two related yet distinctive processes of EED and CCD might help to explain somatization tendency in East Asians. These hypotheses, however, await sustained empirical validation. Therefore, the goal of the current study is to replicate and extend the structural model of somatization, validated in Chinese (Zhou et al., in press), to South Koreans. We hypothesized: (1) that the EED and CCD aspects of somatization would be positively correlated; (2) that EED would be associated with somatic symptom reporting, even when controlling for psychological symptom reporting (i.e., the EED findings are specific to somatic symptoms); and (3) that CCD would be associated with traditional values.

\section{Methods}

\section{Participants}

Psychiatric outpatients were recruited from two sites in South Korea: the psychological clinic of Yonsei University Severance Hospital, located in the metropolitan area of Seoul; and the psychological clinic of Yonsei Wonju College of Medicine, located in Wonju. The study was conducted in accordance with the Helsinki Declaration, 1989 revision.

Potential participants were included in the final analysis if they: (a) had no history of psychosis, mania, or neurocognitive deficits; (b) were between the ages of 18 and 65; (c) had no missing data on self-report measures; and (d) had at least one of the core symptoms of depression or neurasthenia (i.e., depressed mood, anhedonia, or persistent fatigue) during a semi-structured clinical interview (details of the interview procedure can be found in Ryder et al., 2008; information 
obtained from the interview was used to screen participants in the current study). Potential participants consented to participate in the current study and were aware that their participation was completely voluntary.

The final sample comprised 65 men and 144 women, with a mean age of 34 years (range $1 / 419-65, S^{1 / 413}$ ), including 97 out patients from Seoul and 112 outpatients from Wonju. Concerning participants' treatment status: $89 \%$ were currently taking psychiatric medication; $85 \%$ had taken psychiatric medication in the past; $7 \%$ were taking one or more herbal remedies; and $10 \%$ had taken herbal remedies in the past.

\section{Questionnaires}

\section{CPAI-Somatization (CPAI-SOM)}

The somatization subscale of the Chinese Personality Assessment Inventory (CPAI-SOM; Cheung et al., 1996) is a 15-item measure assessing beliefs and practices of somatization when one is distressed. Although the subscale was originally developed to capture a unidimensional construct of somatization, two sub components (i.e., EED and CCD) have been identified in Chinese participants (Zhou et al., in press). Note that in recent years, the Chinese Personality Assessment Inventory has been reconceptualized as the CrossCultural Personality Assessment Inventory (Cheung et al., 2013), and has been validated in a Korean sample (Chon and Cho, 2004).

\section{CPAI-Modernization (CPAI-MOD)}

The Modernization subscale of the Chinese Personality Assessment Inventory (CPAI-MOD; Cheung et al., 1996) is a 15-item measure of modern vs. traditional values in a number of domains, including family relationships, materialism, hierarchical order, rituals, and chastity. The scores reflect individuals' responses to societal modernization in cultures traditionally dominated by Confucian teachings. A higher score indicates a greater adherence to modernization values or a lower endorsement of traditional values, whereas a lower score indicates a greater adherence to traditional values

\section{Symptom questionnaire}

The Symptom Questionnaire consists of an 11-item somatic symptom subscale and a 17-item psychological symptom subscale. The two subscales assess levels of somatic and psychological symptoms of depression. Adequate psychometric and cross-cultural me surement properties have been established (Ryder et al., 2008).

\section{Results}

Structural equation modeling (SEM) was conducted to test the mechanisms of somatization, in relation to levels of so- matic symptoms and traditional values. The parceling technique was used to reduce the variable numbers, so that individual items from the relevant measures were randomly selected and grouped. Following Little and colleagues' recommendation (Little et al., 2002), we constructed three parcels for each latent variable. Maximum likelihood estimation (ML) was used, with the following criteria for acceptable fit (Bryne, 1994, 2012; Ullman, 1996; Hu and Bentler, 1999): non-significant $\chi^{2} ; \chi^{2} / \mathrm{df}$ ratioo $<5$;CFI and TLI $>$.90; RM$\mathrm{SEA}<.10$; and $\mathrm{SRMR}<.08$.

The structural model is shown in Fig. 1. Overall, the model showed good fit: $\chi^{2}(83)^{1 / 4} 164.04, p<.001$; $\chi^{2} / \mathrm{df}^{1} / 41.98 ; \mathrm{CFI} 1 / 4.93 ;$ TLI $1 / 4.91 ; \mathrm{RMSEA}^{1} / 4.07$ (90\% CI from.05 to.08); SRMR $1 / 4.10$. As predicted, somatic symptoms were positively associated with EED, controlling for psychological symptoms. In contrast, lower endorsement of traditional values (or higher endorsement of modernization values) was negatively associated with $\mathrm{CCD}$.

\section{Discussion}

This study is one of the few empirical tests of the mechanisms of somatization. The structural model, originally validated in Chinese patients, was replicated and extended to South Korean psychiatric utpatients. Our findings are consistent with the hypotheses that: (1) the cultural script for depression among South Koreans is composed of at least two correlated aspects; (2) that the EED aspect is associated with somatic symptoms (controlling for psychological symptoms); and (3) that the CCD aspect is associated with cultural values.

The model for South Koreans was largely similar to the one confirmed for Chinese participants (Zhou et al., in press). In both cultural groups, EED and CCD were differently related to symptoms of depression. Specifically, EED—reflecting ways in which people experience and express distress-predicted direct reports of bodily symptoms. In addition to these similarities, some minor differences existed in the link between EED and traditional values. EED had a significant secondary association with traditional values in a previous study of Chinese participants; no such relation was found in the current study. In other words, the proposed model emerged more clearly in the South Korean sample despite being originally developed in a Chinese cultural context.

A notable limitation of the study is that the measure of somatization we used was originally developed to assess a unidimensional construct, and then was separated into two sets of items in order to capture the two proposed aspects of somatization. While these small sets of items have proven sufficient for parceling, and then for testing the proposed model, they are suboptimal for future use as conventional subscales. The field would benefit from the development and validation of measurement tools designed specifically to 
Figure 1

SEM for Korean clinical sample.

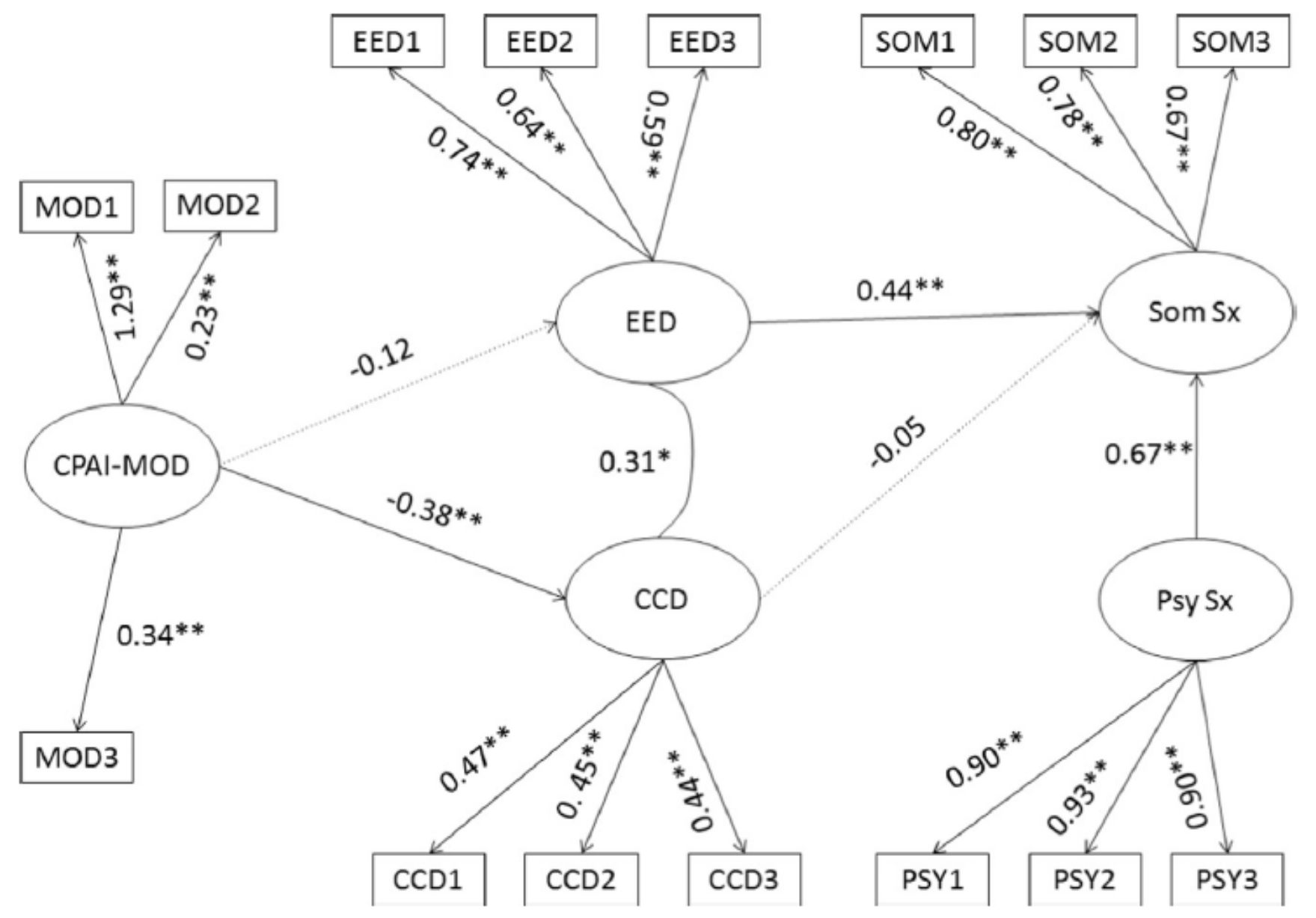

assess cultural scripts of depression. A greater number of items, specifically designed to assess each aspect of somatization, would likely enhance reliability and validity of such a measure.

Moreover, there is a need to move beyond self-report methodologies, as results obtained from self-report questionnaires are subjected to potential response and recall biases. Future studies of cultural scripts of somatization should seek to include multiple methods, such as social experiments, physiological readings, ethnographies, and situation sampling (Doucerain et al., in press; Ryder et al., 2011). Such methods could be used to help promote a greater understanding of the role of Hwa-Byung in depression in South Korea. The inclusion of symptoms of Hwa-Byung, especially anger related symptoms, would extend and generalize our findings to a wider range of Korean patients.

The current study largely replicated a model of East Asian depression previously established in China (Zhou et al., in press), and extended the findings to the South Korean context. Rather than simply assuming that Chinese models will automatically function throughout East Asia, replication in a different East Asian society bolsters our confidence that the model can be used to help explain somatization in these societies more broadly. Taken together, these findings suggest that the tendency to emphasize somatic symptoms of depression can be understood as culturally shaped, where general cultural values are ultimately linked to specific clinical outcomes.

\section{Role of funding source}

Neither the Canadian Institutes of Health Research nor any other funding source played any role in the conduct of the research or the preparation of this manuscript.

\section{Conflict of interest}

The authors have no conflicts of interest to report. 


\section{Acknowledgments}

The authors gratefully acknowledge the assistance of Michael Bagby, Jessica Dere, Shuqiao Yao, and Xiongzhao Zhu for their contributions to setting up and coordinating the multinational study from which these data were obtained. This research was supported by a standard operating grant from the Canadian Institutes of Health Research (MOP 82871) to AGR. Preparation of this manuscript by Xiaolu Zhou was sponsored by the Shanghai Pujiang Program (14PJC087).

\section{References}

Bryne, B.M., 1994. Structural Equation Modeling With EQS and EQS/Windows: Basic Concepts, Applications, and Programming. Sage Publications, Thousand Oaks, CA.

Bryne, B.M., 2012. Structural Equation Modeling With Mplus: Basic Concepts, Applications, and Programming. Routledge, New York.

Cheung, F.M., Leung, K., Fan, R., Song, W.Z., Zhang, J.X., Zhang, J.P., 1996. Development of the Chinese Personality Assessment Inventory (CPAI). J. Cross-Cult. Psychol. 27, 181-199.

Cheung, S.F., Cheung, F.M., Fan, W., 2013. From Chinese to Cross-Cultural Personality Inventory: a combined emic-etic approach to the study of personality in culture. In: Gelfand, M.J., Chiu, C., Hong, Y. (Eds.), Advances in Culture and Psychology, vol. 3. Oxford University Press, Oxford, pp. 117-180.

Chon, K. K., Cho, J. R., 2004. Validation studies of CPAI-2 in Korea In: Proceedings of the XXVIII International Congress of Psychology, Beijing, China.

Dere, J., Sun, J., Zhao, Y., Persson, T.J., Zhu, X., Yao, S., Bagby, R.M., Ryder, A.G., 2013. Beyond "somatization" and "psychologization": Symptom-level variation in depressed Han Chinese and Euro-Canadian outpatients. Front. Psychol. 4, 377-390.

Doucerain, M., Vargas, S., Ryder, A.G., 2015. Mixedmethods research: integrating qualitative and quantitative approaches to the psychological study of culture. In: Zane, N., Bernal, G., Leong, F. (Eds.), Culturally-Informed EvidenceBased Practice for Ethnic Minorities: Challenges and Solutions. American Psychological Association, Washington, DC (in press).

Hu, L., Bentler, P.M., 1999. Cutoff criteria for fit indexes in covariance structure analysis: Conventional criteria versus new alternatives. Struct. Equ. Model. 6, 1-55.

Keyes, C.L.M., Ryff, C.D., 2003. Somatization and mental health: a comparative study of the idiom of distress hypothesis. Soc. Sci. Med. 57, 1833-1845.

Kim, K.I., 1973. Traditional concepts of disease in Korea. Korea J. 12, 12-18.
Kim, K.I., Li, D., Kim, D.H., 1999. Depressive symptoms in Koreans, Korean-Chinese and Chinese: a transcultural study. Transcult. Psychiatry 36, 303-316.

Kim, K.L., 1999. Culture and illness behavior in South Korea. Transcult. Psychiatry 36, 65-77.

Kim, K.L., Rhi, B.Y., 1976. A review of Korean cultural psychiatry. Transcult. Psychiatry 13, 101-114.

Kim, M.T., 2002. Measuring depression in Korean Americans: development of the Kim depression scale for Korean Americans. J. Transcult. Nurs. 13, 109-117.

Kirmayer, L.J., Robbins, J.M., 1991. Introduction: concepts of somatization. In: Kirmayer, L.J., Robbins, J.M. (Eds.), Current Concepts of Somatization: Research and Clinical Perspectives. American Psychiatric Press, DC, pp. $1-19$.

Kleinman, A., 1982. Neurasthenia and depression: a study of somatization and culture in China. Cult. Med. Psychiatry 6, 117-190.

Kleinman, A., 1986. Social Origins of Disease and Distress: Depression, Neurasthenia, and Pain in Modern China. Yale University Press, New Haven, CT.

Lin, K.M., Lau, J.K.C., Yamamoto, J., Zheng, Y.P., Kim, H.S., Cho, K.H., Nagasaki, G., 1992. Hwa-Byung: a community study of Korean Americans. J. Nerv. Ment. Dis. 180, 386-391.

Little, T.D., Cunningham, W.A., Shahar, G., 2002. To parcel or not to parcel: exploring the question, weighing the merits. Struct. Equ. Model. 9, 151-173.

Pang, K.Y.C., 1998. Symptoms of depression in elderly Korean immigrants: narration and the healing process. Cult. Med. Psychiatry 22, 93-122.

Pang, K.Y.C., 2000. Symptom expression and somatization among elderly Korean immigrants. J. Clin. Geropsychol. 6, 199-212.

Park, S.Y., Bernstein, K.S., 2008. Depression and Korean American immigrants. Arch. Psychiatr. Nurs. 22, 12-19.

Park, S.Y., Cho, S., Park, Y., Bernstain, K.S., Shin, J.K., 2013. Factors associated with mental health service utilization among Korean American immigrants. Community Ment. Health J. 49, 765-773.

Park, Y.J., Kim, H.S., Kang, H.C., Kim, J.W., 2001. A survey of Hwa-Byung in middle age Korean women. J. Transcult. Nurs. 12, 115-122.

Parker, G., Cheah, Y.C., Roy, K., 2001. Do the Chinese somatize depression? A cross cultural study. Soc. Psychiatry Psychiatr. Epidemiol. 36, 287-293.

Ryder, A.G., Ban, L.M., Chentsova-Dutton, Y.E., 2011. Towards a cultural-clinical psychology. Soc. Personal. Psychol. Compass 5, 960-975.

Ryder, A.G., Chentsova-Dutton, Y.E., 2012. Depression in cultural context: "Chinese somatization", revisited. Psychiatr. Clin. N. Am. 35, 15-36.

Ryder, A.G., Yang, J., Zhu, X., Yao, S., Yi, J., Heine, S.J., 
Bagby, R.M., 2008. The cultural shaping of depression: somatic symptoms in China, psychological symptoms in North America? J. Abnorm. Psychol. 117, 300-313.

Simon, G.E., VonKorff, M., Piccinelli, M., Fullerton, C., Ormel, J., 1999. An international study of the relation between somatic symptoms and depression. N. Engl. J. Med. $341,1329-1335$.

Suh, S., 2013. Stories to be told: Korean doctors between Hwa-byung (Fire-illness) and depression, 1970-2011. Cult. Med. Psychiatry 37, 81-104.

Ullman, J.B., 1996. Structural equation modeling. In: Tabachnick, B.G., Fidell, L.S. (Eds.), Using Multivariate Statistics, 3rd ed. Harper Collins, New York, pp. 708-819.

Yoo, S.K., 2001. The cultural impact on depression expression and attitudes toward seeking professional help: a comparative study of Americans and South Koreans. Asia Pac. Educ. Rev. 2, 94-100.

Yoo, S.K., Skovhot, T.M., 2001. Cross-cultural examination of depression expression and help-seeking behavior: a comparative study of American and Korean college students. J. Coll. Couns. 4, 10-19.

Zhou, X., Dere, J., Zhu, X., Yao, S., Chentsove-Dutton, Y.E., Ryder, A.G., 2011. Anxiety symptom presentations in Han Chinese and Euro-Canadian outpatients: is distress always somatized in China? J. Affect. Disord. 135, 111-114.

Zhou, X., Peng, Y., Zhu, X., Yao, S., Dere, J., ChentsoveDutton, Y.E., Ryder, A.G., 2015. From culture to symptom: testing a structural model of 'Chinese Somatization'. Transcult. Psychiatry, in press. 